\title{
RECOGNIZING MENTAL ROTATION AND SPATIAL VISUALIZATION SKILLS IN MINECRAFT IN-GAME PRACTICES
}

\author{
Syquia SMS* \\ Xavier School San Juan, Philippines
}

\begin{abstract}
The video game Minecraft has been acknowledged by several researchers and educators as a potential tool for learning spatial skills. However, there exists a dearth of research and data on how individuals can recognize such skills in multiple manifestations from in-game practices. Thus, the researcher analyzed the question design, format, and underlying skills in the most widely-used spatial tests that measure mental rotation and spatial visualization, particularly the Differential Aptitude Test: Space Relations (DAT:SR), Paper Folding Test, 3-Dimensional Cube (3DW), Mental Rotation Test (MRT), and Purdue Spatial Visualization Tests: Visualization of Rotations (PSVT:R). Mental rotation refers to the ability to rotate mental images of two-dimensional and threedimensional objects while spatial visualization refers to the ability to recognize and mentally manipulate two-dimensional and three-dimensional objects. After the researcher examined the details of each test, parallel spatial skills that are also evident in Minecraft in-game practices were identified. Then, the researcher determined and analyzed these specific in-game practices by searching for objects within Minecraft that players commonly encounter while playing the game. To illustrate the comparison between the skills in each of these five tests and the in-game practices, the researcher adapted some official question types from each test and took pictures of gameplay with scenarios that display evidence of such practces and skills. Given the ability of a Minecraft player to manipulate the movements and perspectives of their character, and break or place blocks, the researcher deduced that mental rotation and spatial visualization skills can all be recognized from in-game engagements in Minecraft.
\end{abstract}

Keywords: minecraft, spatial, visualization, rotation, tests, skills

\section{Introduction}

\section{Background}

As new activities and technologies grow reliant on creating mental representations of objects, spatial skills have become more critical for one's success (Benbow et al., 2010). Spatial abilities, defined as the capacity to mentally generate, rotate, and transform images, are used to solve many problems in everyday life and various academic disciplines. Daily life tasks that involve spatial skills range from navigating to packing luggage. Similarly, these skills are applied in fields like engineering, graphic design, art, and architecture. Hence, any individual needs to develop their spatial ability.

\section{Significance of Study}

A lack of spatial skills is detrimental to one's understanding of an object's dimensions, orientations, and locations. These skills play a key role in technical fields such as sciences, engineering, 
mathematics, and computer science. Thus, spatial ability must not be neglected among practices in education, especially for students aiming to enter these academic fields (Benbow et al., 2010). Fortunately, there are many specific activities that have been proven to sharpen these skills. One of these activities includes the popular sandbox video game Minecraft, which has been recognized as a space to improve one's mental rotation and spatial visualization. The game's absence of a plot drives players to do anything with the tools that they have access to in-game. Players can also manipulate parts of their world and build structures by breaking or placing blocks, as well as moving their characters along different angles and directions (Nguyen, 2016). Hence, exploring Minecraft as a tool to learn spatial skills can help many, especially children and teenagers, who commonly engage in the game today.

\section{Scope and Limitations}

The objectives of this research paper are to identify, analyze, and compare the manifestations of mental rotation and spatial visualization skills between Minecraft in-game practices and five common spatial tests. These tests include the DAT:SR, Paper Folding Task, 3DW, MRT, and PSVT:R. Conversely, the limitations of this paper are that it does not justify whether Minecraft is the best tool for learning these skills nor prove how the game teaches the skills.

\section{Literature Review}

The researcher will display related works that focus on the importance of spatial skills, the various commonly-used spatial tests, and how the video game Minecraft is currently being utilized as a learning tool. This study aims to analyze how Minecraft in-game can train students' spatial skills.

\section{A Computational Model for Reasoning About the Paper Folding Task Using Visual Mental Images}

The research aims to introduce the mechanics of the paper folding task, a test that assesses one's spatial reasoning skills. It also presents a computational model that demonstrates the solution to this task through visual representations and operations. (Ainooson \& Kunda, 2017).

\section{$\underline{\text { Spatial Reasoning in Minecraft: An Exploratory Study of In-Game Spatial Practices }}$}

The research identifies various Minecraft in-game practices that display spatial reasoning skills through middle school students' gameplay. Its purpose is to explore how these spatial skills can be developed in game-based learning environments (Bar-El \& Worsley, 2020).

\section{$\underline{\text { Recognizing Spatial Intelligence }}$}

This article introduces spatial ability as one of the three key cognitive abilities alongside verbal and quantitative skills. It also defines spatial ability as the capacity to mentally generate, rotate, and transform visual images. This text also cites various studies that found a positive relationship between strong spatial abilities and interest in technical fields that involve manipulating objects. (Benbow et al., 2010). 


\section{Investigating the Relationship Between Spatial Skills and Computer Science}

The research aims to survey current literature about spatial skills and propose a relationship between computer science and spatial skills. It also introduces various tests such as the $2 \mathrm{D}$ mental rotation, PSVT:R, and the Cube Comparison Test (Cutts \& Parkinson, 2018).

Psychometric Properties of the Revised PSVT:R for Measuring First-Year Engineering Students' Spatial Ability

The research aims to investigate the validity and functions of the Revised PSVT:R test in predicting the success of First Year Engineering (FYE) students in their field. It also aims to evaluate the correlations between the Revised PSVT:R and other academic variables (Imbrie et al., 2013).

Playing with Virtual Blocks: Minecraft as a Learning Environment for Practices and Research

This research aims to describe how Minecraft is currently being used in the classroom. It specifically focuses on how Minecraft gameplay can be relevant in influencing a child's development in STEM skills (Lane \& Yi, 2017).

Studying the Impact of Spatial Involvement on Training Mental Rotation with Minecraft

This research aims to analyze the different levels of spatial involvement within Minecraft. Its objective is to demonstrate how various aspects of Minecraft gameplay impact and train one's mental rotation skills (Nguyen, 2016).

\section{Synthesis}

The references in this literature review all contribute to the study that explores the spatial visualization and mental rotation skills that can be developed through Minecraft in-game practices. The information gathered can be summarized into three main points that (1) Minecraft in-game practices can be a potential tool to train spatial skills, (2) spatial skills are vital for a students' success in technical fields, and (3) certain spatial reasoning tests are commonly-used to assess these skills.

The first point was proven through multiple research that analyzed the different levels of spatial involvement. These studies have also identified certain in-game practices that influence children's STEM and spatial reasoning skills.

The second point was proven through an article that defined spatial ability, which is one's capacity to manipulate visual images. The same article also cited various studies to argue that having strong spatial skills shows a great interest in manipulating objects, which is integral to many technical fields.

The third point was proven through research that introduced spatial reasoning tests such as the Revised PSVT:R, Cube Comparison Test, and Paper Folding Task. These tests all assess various components of a students' spatial ability, particularly mental rotation and spatial visualization. 


\section{Literature Gap}

Research has been done about how Minecraft is an effective digital game-based learning tool for skills in mathematics, engineering, biology, and computer science (Lane \& Yi, 2017). There is little research on the relationship between Minecraft and spatial reasoning by testing it on human beings. However, research still has yet to draw clear parallels that illustrate how this game teaches the same skills assessed in spatial ability tests. Thus, the researcher chose to continue this study, exploring the correlation between the skills manifested in Minecraft in-game practices and those assessed spatial tests.

\section{Methodology}

The data collection method that the researcher used is qualitative analysis. This was done by first obtaining official sample questions of each spatial skills test, then building and finding objects in Minecraft that mimic or look similar to the shapes presented in the tests. Next, the researcher captured various screenshots of these objects in the game that corresponded to the tests. After compiling the images of the spatial skills test questions and Minecraft structures built, the researcher drew parallels between each test and the in-game practices such as the player movements or building skills used for the objects in the screenshots.

\section{Results and Discussion}

\section{Spatial Visualization Tests}

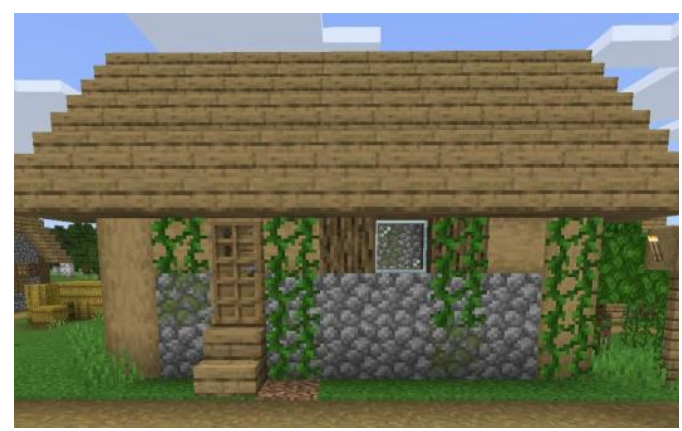

Figure 1: Front view of a house

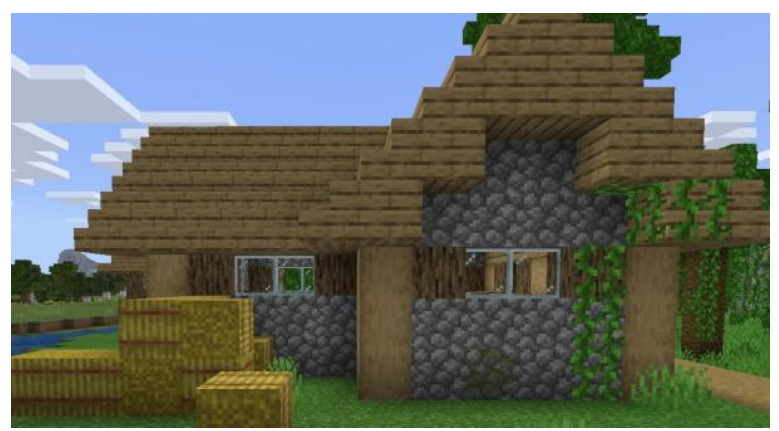

Figure 2: Side view of a house

Spatial visualization is defined as one's ability to mentally manipulate two-dimensional and threedimensional objects. The spatial tests that assess spatial visualization are the DAT:SR and the Paper Folding Task. In the context of Minecraft, this skill is commonly used when players build structures as they would have to visualize the size and appearance of their output while building (Bar-El \& Worsley, 2020). As seen in Figures 1 and 2, the different views of a house and perspectives allow players to mentally map these objects in their heads. Similarly, they can tell how objects look on each side by estimating their sizes in terms of blocks. 


\section{Differential Aptitude Test: Spatial Relation (DAT:SR)}

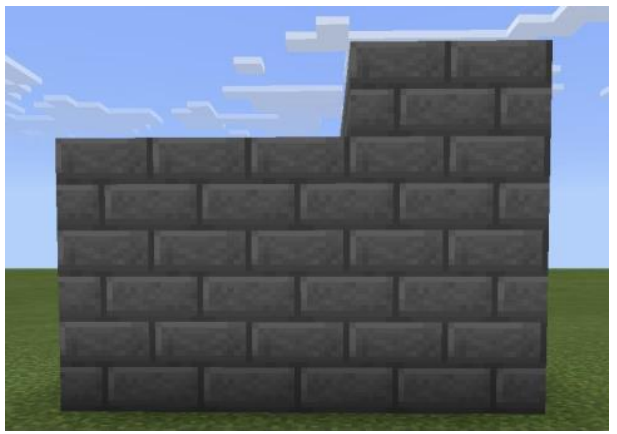

Figure 3: Side view of a structure

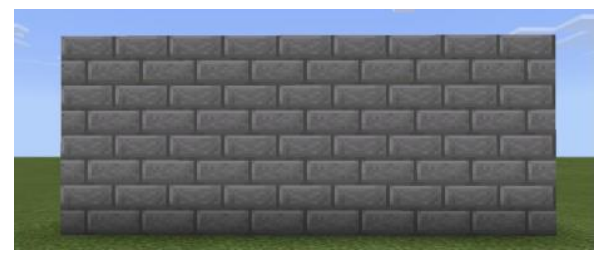

Figure 5: Back view of the structure

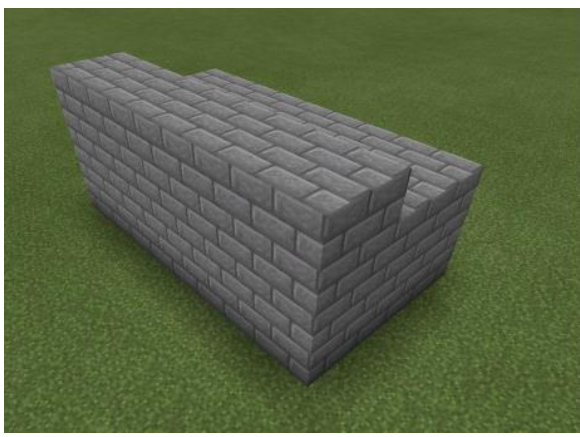

Figure 4: Isometric view of a structure

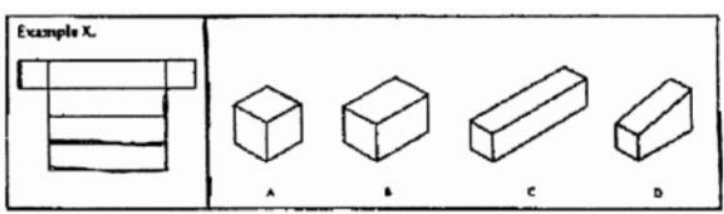

Figure 6: DAT:SR

The first test is the DAT:SR, which prompts test-takers to choose a letter choice out of four on which folded object is the outcome of an unfolded shape when folded as in Figure 6 (Imbrie et al., 2013). This is a test of spatial visualization because one would have to estimate the sizes of the faces of the unfolded object. After which, they must be able to mentally imagine the unfolded shape folding to accurately form the folded object.

In Minecraft, players establish the lengths, widths, and heights of 3-Dimensional (3D) objects that they build in-game like in Figure 4. They can count the number of blocks as units of measurement on each side to tell if the object will turn out as expected. In addition, while players are building, they can see each side of the structure as in Figure 3 and 5. Since players estimate the measurement of the 2D sides of their builds in terms of the number of blocks, they are trained to estimate if certain faces of unfolded objects as tested in the DAT:SR would fit with each other to form a 3D object. 


\section{Paper Folding Task}

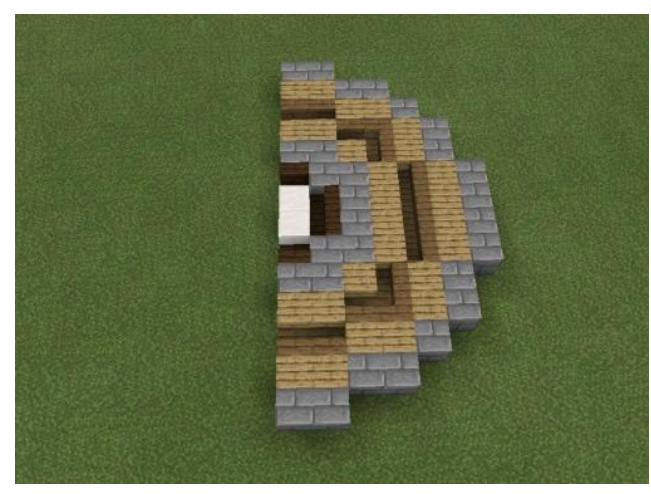

Figure 7: Half part of a pattern

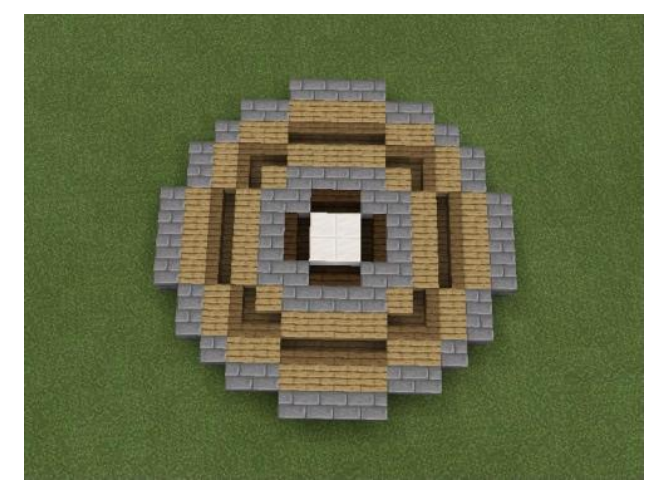

Figure 8: Full pattern

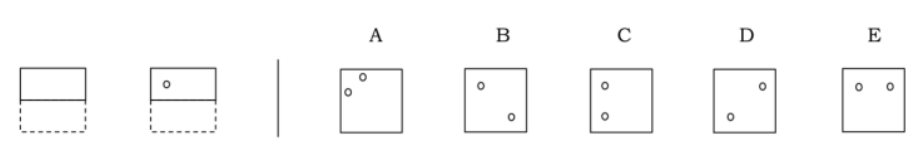

Figure 9: VZ-2 Paper Folding Test

The second test is the Paper Folding Task, which prompts test-takers to choose a letter choice of what a paper with a print folded in half looks like when the other half is unfolded as in Figure 9 (Ainooson \& Kunda, 2017). This is a test of spatial visualization because one would have to mentally map how a print looks when mirrored and made into a symmetrical image.

Again, in Minecraft, players establish how large certain parts of objects are especially when building patterns. These patterns often involve symmetry, which trains players to build the first half of their structure as in Figure 7 and 8. Then, they create the other half of it by mirroring the first so that they can complete the pattern as shown in Figure 8. Through creating patterns or structures in Minecraft, players are trained to visualize the outcome of their build just by mentally manipulating and mirroring the object as tested in the Paper Folding Task while imagining it as the other half of the build. 


\section{Mental Rotation Tests}

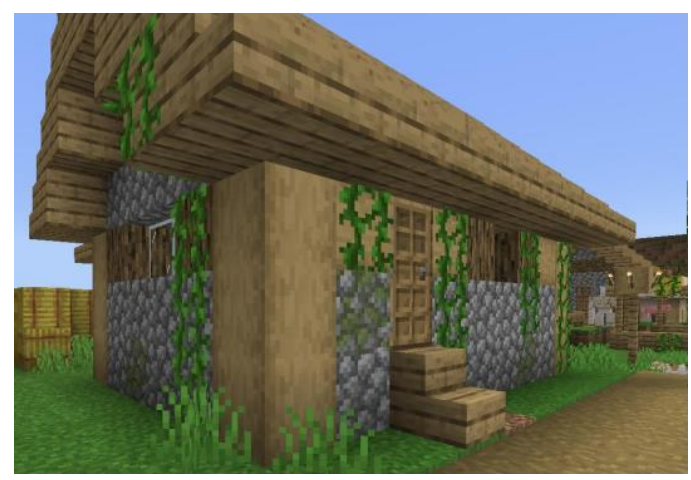

Figure 10: Front trimetric view of a house

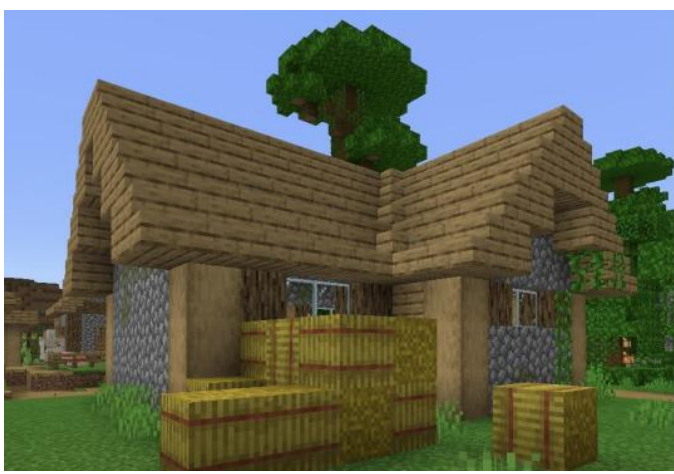

Figure 11: Side-angled view of a house

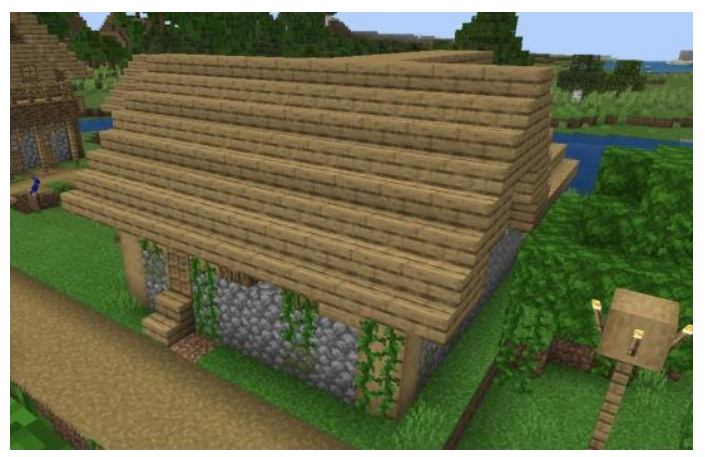

Figure 12: Upper trimetric view of a house

Mental rotation is defined as one's ability to rotate mental images of two-dimensional and threedimensional objects. The spatial tests that assess spatial visualization are the 3DW, MRT, and the PSVT:R. In the context of Minecraft, this skill is commonly used when players build 3D objects, as they can view the different angles of the same object they built (Worsley \& Bar-El, 2020). As seen in Figures 10,11, and 12, a house's different trimetric and angles allow players to mentally rotate these objects. Similarly, they can imagine how the objects look on each side by recognizing the other perspectives of the same object. 


\section{3-Dimensional Cube (3DW)}

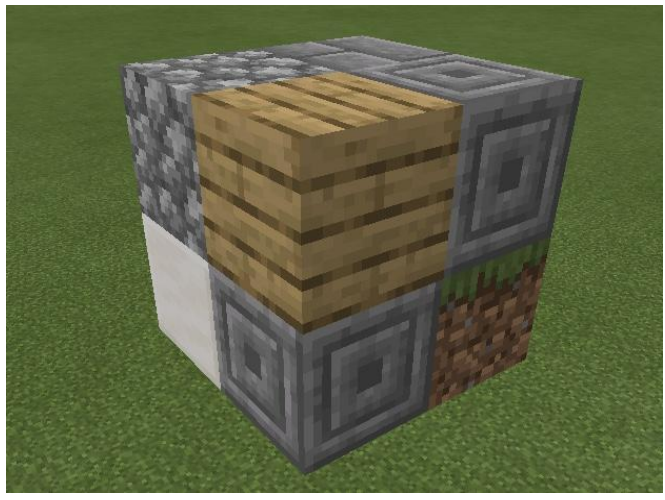

Figure 13: Upper trimetric view of a cube

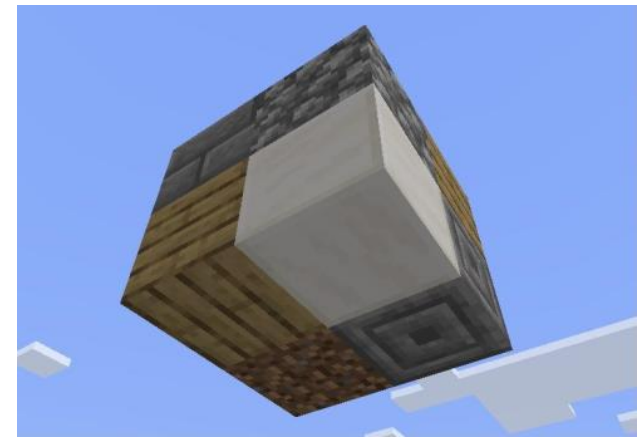

Figure 15: Lower-left trimetric view of a cube

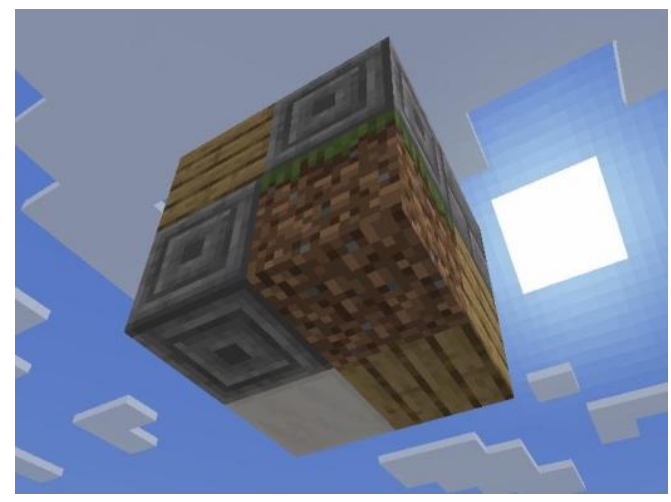

Figure 14: Lower-right trimetric view of a cube

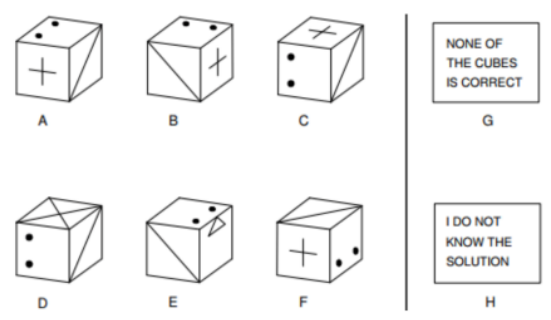

Figure 16: 3DW (Cube Comparison Test)

The third test is the 3DW, which prompts test-takers to choose a letter choice out of six similarlooking cubes on which cube is the same as the given one in a different orientation as in Figure 16 (Cutts \& Parkinson, 2018). However, the cube is in cavalier view meaning that only three faces of each cube choice are revealed. Thus, this is a test of mental rotation as it tests one's capacity to relate each face of the cubes to its neighbors to decide which one corresponds to the original cube.

In Minecraft, players can go around objects and rotate character's views of them, getting multiple angled perspectives. Building in-game requires players to use perspective to complete their 3D structures from all possible views of it as seen in Figures 13, 14, and 15. Through creating 3D objects in Minecraft, players are trained to visualize their build's looks from any view and mentally rotate it as tested in 3DW as long as they can see one of the angled perspectives of the object. 


\section{Mental Rotations Test (MRT)}

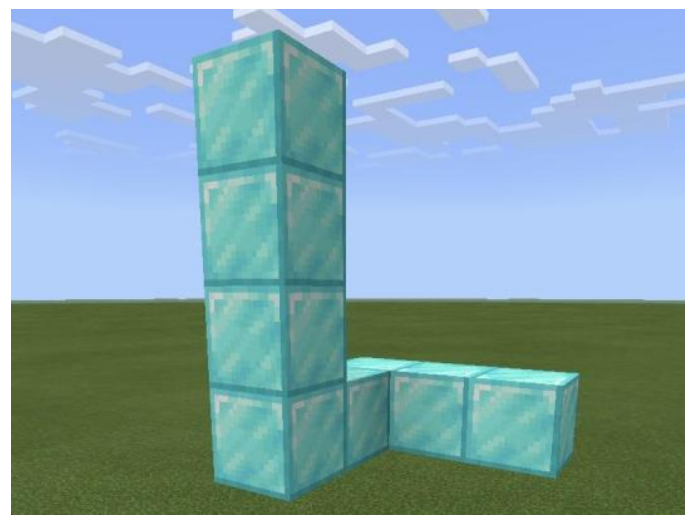

Figure 17: Left trimetric view of MRT shape

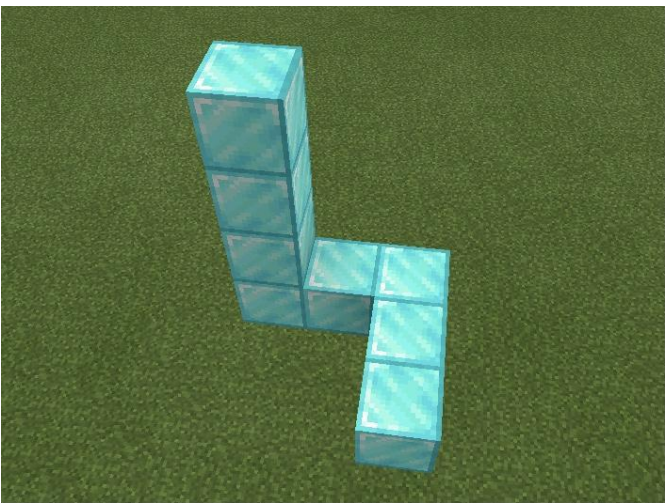

Figure 19: Upper trimetric view of MRT shape

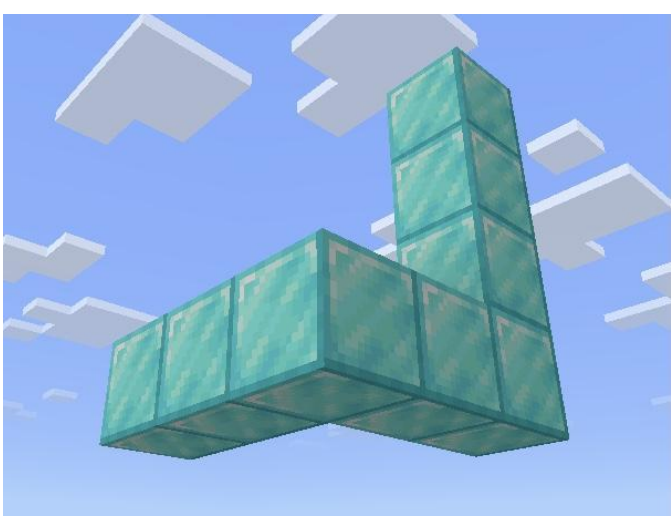

Figure 18: Lower trimetric view of MRT shape
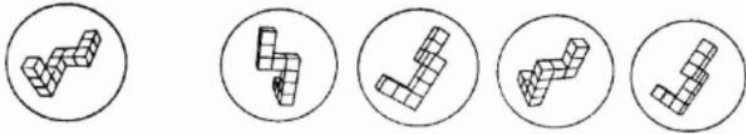

Figure 20: Mental Rotations Test

The fourth test is the MRT, which prompts test-takers to choose a choice out of four similar-looking shapes made of cubes on which one is the same as the given one in a different orientation as in Figure 20 (Imbrie et al., 2013). Thus, this is a test of mental rotation as it tests one's capacity to mentally rotate and count the cubes in each choice to decide which one corresponds to the original one.

Again, in Minecraft, players can go around objects and rotate their character's views of them. Similarly, they can estimate the length of certain parts of these objects by counting the blocks. Building in-game requires players to view their objects from different perspectives as seen in Figures 17, 18, and 19. By creating Minecraft objects, players are trained to estimate the size of their builds and can mentally rotate their build from any view as tested in the Mental Rotations Test. 


\section{Purdue Spatial Visualization Tests: Visualization of Rotations (PSVT:R)}

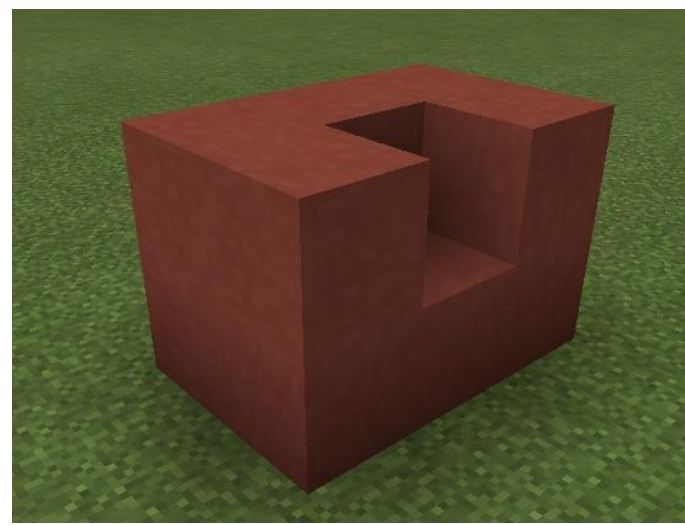

Figure 21: Left trimetric view of the shape

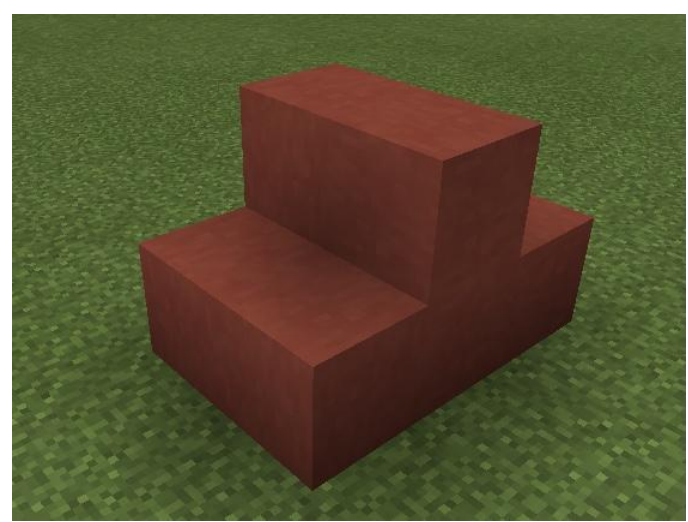

Figure 23: Left trimetric view of second shape

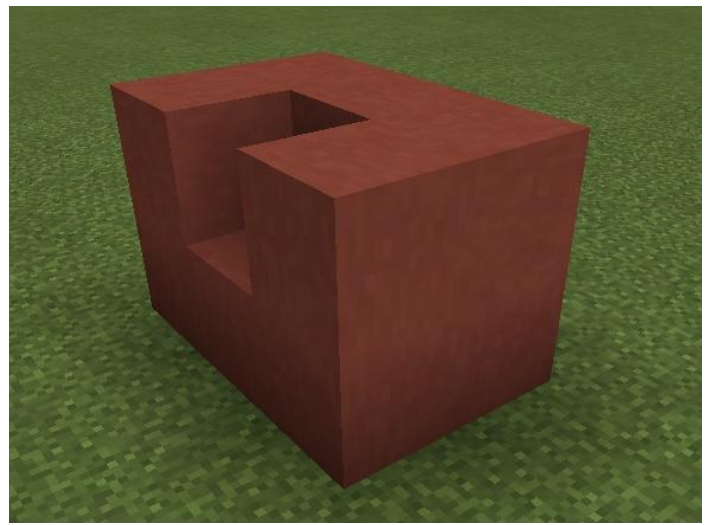

Figure 22: Right trimetric view of the shape

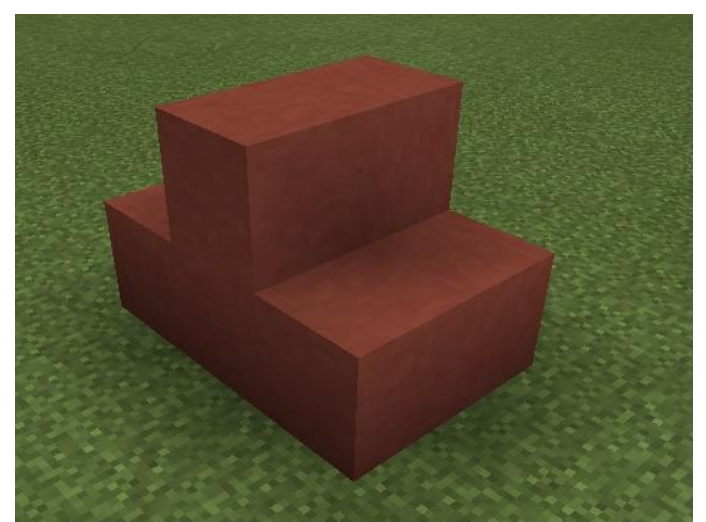

Figure 24: Right trimetric view of second shape

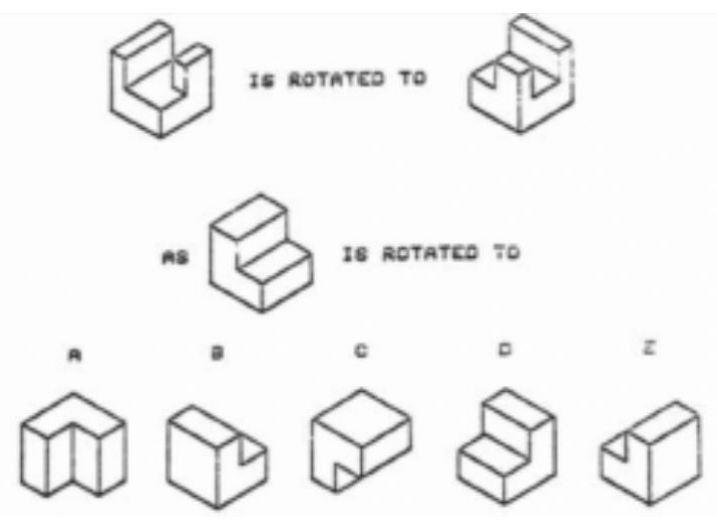

Figure 25: PSVT:R

The fifth test is the PSVT:R, which prompts test-takers to choose a letter choice out of five that would show how a different object would look when rotated in the same way that a different object is rotated as in Figure 25 (Imbrie et al., 2013). Thus, this is a test of mental rotation as it tests one's capacity to take the representation of a certain object when rotated and apply that same rotation to another object. 
Again, in Minecraft, players can go around objects and rotate their character's views of them. Going around different objects in-game helps players learn how they would appear in various orientations and rotations as seen in Figures 21, 22, 23, and 24. Through viewing objects from multiple perspectives in Minecraft, players are trained to mentally rotate builds and thus apply similar rotations to other ones as tested in the PSVT:R.

\section{Conclusion}

All of the figures and practices in Minecraft have shown similar skills to those that are assessed in each spatial test. Given the traits of Minecraft including player movement, 3-Dimensional worlds, and building structures in-game, playing the game can indeed sharpen the skills of spatial visualization and mental rotation. Firstly, the DAT:SR and Paper Folding Task tests that assess spatial visualization are evident in the practices of viewing different faces of an object in-game and estimating or measuring the sizes or lengths of their sides in terms of the number of blocks. Secondly, the 3DW, MRT, and PSVT:R tests that assess mental rotation are seen in the in-game practices by traveling around objects, rotating the character's views, and getting angled perspectives from any of their sides. Hence, spatial visualization and mental rotation skills that are assessed in widely-used spatial skills tests correlate with Minecraft in-game practices.

\section{References}

Ainooson, J. \& Kunda, M. (2017). A Computational Model for Reasoning About the Paper Folding Task Using Visual Mental Images. Cognitive Science. https://cogsci.mindmodeling.org/2017/papers/0298/paper0298.pdf

Bar-El, D. \& Worsley, M. (2020). Spatial Reasoning in Minecraft: An Exploratory Study of In-game Spatial Practices. In M. Gresalfi \& I. S. Horn (Eds.), $14^{\text {th }}$ International Conference of the Learning Sciences: The Interdisciplinarity of the Learning Sciences, ICLS 2020 - Conference Proceedings (pp. 709-712). International Society of the Learning Sciences. https://www.scholars.northwestern.edu/en/publications/spatial-reasoning-in-minecraft-anexploratory-study-of-in-game-sp

Benbow, C., Park, G., \& Lubinski, D. (2010, Nov. 2). Recognizing Spatial Intelligence. Scientific American. https://www.scientificamerican.com/article/recognizing-spatial-intel/

Cutts, Q. \& Parkinson, J. (2018). Investigating the Relationship Between Spatial Skills and Computer Science. Proceedings of the 2018 ACM Conference on International Computing Education Research, 106-114. https://doi.org/10.1145/3230977.3230990

Imbrie, P., Kim-Kang, G., Maeda, Y., \& Yoon, S. (2013). Psychometric Properties of the Revised PSVT:R for Measuring First Year Engineering Students' Spatial Ability. International Journal of Engineering Education, 29(3), 763-776. https://www.researchgate.net/publication/261760571_Psychometric_Properties_of_the_Revis ed_PSVT_R_for_Measuring_First_Year_Engineering_Students\%27_Spatial_Ability

Lane, H. C. \& Yi, S. (2017). Playing with Virtual Blocks: Minecraft as a Learning Environment for Practice and Research. Cognitive Development in Digital Contexts, 145-166. https://doi.org/10.1016/b978-0-12-809481-5.00007-9

Nguyen, A. \& Rank, S. (2016). Studying the Impact of Spatial Involvement on Training Mental Rotation with Minecraft. Proceedings of the 2016 CHI Conference Extended Abstracts on Human Factors in Computing Systems, 1966-1972. https://doi.org/10.1145/2851581.2892423 